\title{
Coexistence in Exotic Scenarios of a Modified Abrams-Strogatz Model
}

\author{
RENATO COLUCCI, ${ }^{1}$ JORGE MIRA, ${ }^{2}$ JUAN J. NIETO, ${ }^{3,4}$ AND M. VICTORIA OTERO-ESPINAR ${ }^{3}$ \\ ${ }^{1}$ Department of Mathematical Sciences, Xi'an Jiatong-Liverpool University, Suzhou, China; ${ }^{2}$ Departa- \\ mento de Física Aplicada, Universidade de Santiago de Compostela, 15782 Santiago de Compostela, \\ Spain; ${ }^{3}$ Departamento de Análise Matemática, Instituto de Matemáticas, Universidade de Santiago de \\ Compostela, 15782, Santiago de Compostela, Spain; and ${ }^{4}$ Department of Mathematics, Faculty of \\ Science, King Abdulaziz University, Jeddah, Saudi Arabia
}

Received 4 July 2014; accepted 6 October 2014

\begin{abstract}
We work on a model that has succeeded in describing real cases of coexistence of two languages within a closed community of speakers, taking into account bilingualism and incorporating a parameter to measure the distance between languages. The dynamics of this model depend on a characteristic exponent, which weighs the power of the size of a group of speakers to attract new members. So far, this model had been solved only when this characteristic exponent is greater than 1. In this article, we have managed to solve the nature of the stability of all the possible situations for this characteristic exponent, that is, when it is less or equal than 1 and covering also the situations produced when it is 0 or negative. We interpret these new situations and find that, even in such exotic scenarios, there are configurations of the resulting societies where all the languages coexist. (C) 2014 Wiley Periodicals, Inc. Complexity 21: 86-93, 2016
\end{abstract}

Key Words: language competition; bilingualism; nonlinear dynamics; language modeling; social physics; physics and society

\section{INTRODUCTION}

anguage diversity, one of the best samples of human intelligence, has been the subject of scientific work from many points of view, from cognitive sciences [1] (see also [2]) to physics and mathematics [3, 4]. This rising field of language dynamics has even interesting connections with unexpected fields such as genomes [5] and biology [6].

Correspondence to: Jorge Mira, E-mail: jorge.mira@usc.es
The approaches include debates on the formation, development, and dissemination of languages. Dissemination implies that, in a given moment, two languages enter in contact, that is: groups of human beings speaking different contact each other (see, e.g., [7] for a general discussion on agent-based models in social sciences). This enriches both communities but, in several cases, it is the source of a conflict, being at the center of the political agenda in many places of the world.

It is therefore of interest to pose questions like: what is the fate of a community exposed to the influence of two 
languages? Is it possible to arrive to a point of stable linguistic coexistence in such communities? In this context, a paper by Abrams and Strogatz [8] introduced an important novelty: the treatment of the problem in a global mean field perspective (see [9] for an accurate review about the topic), instead of focusing on independent speakers. In this way, they arrived to a minimal model in which the probability for speakers to scape from one language $X$ to another $Y$ is proportional to a parameter of status $s$ and to the size of the group of speakers of language $Y$. To weigh the role of the group size, they introduced an exponent $a$.

In detail, they consider two monolingual populations $x$ and $y$ (that speak $X$ and $Y$, respectively), such that $x+y=1$, and introduce the probabilities $P_{x y}(x, s)$ and $P_{y x}(x, s)$ for shifts from $X$ to $Y$ and viceversa:

$$
P_{y x}(x, s)=c x^{a} s, P_{x y}(x, s)=c(1-x)^{a}(1-s),
$$

where the parameter $s \in[0,1]$ measures the relative social status of one language with respect to the other and represents a criterion to select the language that gives more social and personal opportunities.

Then, the final model can be written as:

$$
\dot{x}=y P_{y x}(x, s)-x P_{x y}(x, s) .
$$

With such minimal model, they succeeded in fitting the historical evolution of the relative fractions of speakers of two languages in contact across many different regions of the world. From fits of the historical data to their differential equation, they could obtain the status perceived for each of the languages, as well as the exponent $a$. Surprisingly, it was found that it is quite constant along different cultures $(a=1.31 \pm 0.25)$, which eventually might be viewed as the mark of a universal trait of human relations. The value $a>1$ seems reasonable, as it suggests that the size of a group is a major factor of attraction, as expected in social animals like humans.

But, another conclusion of [8] was that the competition of two languages for speakers in a given society inevitably leads to the extinction of one of them. Researchers have addressed the extinction of languages as a huge problem of our century: many languages will face extinction in a short term, while even quite widely spoken languages may be in danger in the long term (see [10]). Another interesting aspect is that the languages death appears to be intrinsically related to the collapse of the ecosystems, such as rain forests, and as a consequence the maintenance of cultural diversity is tied to that of ecological diversity (see, e.g., [11]). However, this does not seem to be the case of some places in the world, where the competition has given rise to the coexistence of the two languages and the appearance of a bilingual population (see [12-14] for a discussion on the role of bilinguals).
Inspired by this and by the intuition that similarity between languages could help to the natural birth of bilingual groups, Mira and Paredes [15] modified the AbramsStrogatz model introducing the possibility of bilingualism and a coefficient of similarity (or, inversely, distance between languages), that could also be used to define a metric of a space of languages (it is worth mentioning that other models have been proposed to describe case of coexistence, see for instance [16-18]).

This model considers three populations (two monolinguals, $x$ and $y$, and one bilingual, $b$ ) and a new parameter (besides $a$ and $s$ ), denoted by $k$, which gives a measure of the similarity between the two languages. The transition probabilities are represented then by the following functions

$$
\begin{aligned}
& P_{X B}=c \cdot k(1-s)(1-x)^{a}, \\
& P_{Y B}=c \cdot k s(1-y)^{a}, \\
& P_{B X}=P_{Y X}=c \cdot(1-k) s(1-y)^{a}, \\
& P_{B Y}=P_{X Y}=c \cdot(1-k)(1-s)(1-x)^{a},
\end{aligned}
$$

and the system for the three populations $x, y$, and $b$ becomes

$$
\begin{aligned}
& \dot{x}=y P_{Y X}+b P_{B X}-x\left(P_{X Y}+P_{X B}\right), \\
& \dot{y}=x P_{X Y}+b P_{B Y}-y\left(P_{Y X}+P_{Y B}\right), \\
& \dot{b}=x P_{X B}+y P_{Y B}-b\left(P_{B Y}+P_{B X}\right) .
\end{aligned}
$$

This approach permitted the fit of historical data of the Autonomous Community of Galicia (northwest Spain), but it opened also the exploration of different scenarios resulting from the system of equations (3) depending on their parameters. In this line, computer simulations showed that, for $a=1.31$ and certain ranges of the parameters $k$ and $s$, there are stable points of equilibrium without extinction of any of the languages [19]. Later, the model was solved analytically [20], to define the existence and nature of its equilibrium points for $a>1$, which covers the situations described by the different sets of real data that have been successfully fitted so far. Complementing this, Colucci et al. [submitted] found explicitly the region for the parameters $(k, s)$ in which there exist coexistence solutions using bifurcation theory in the case $a \in(1,2)$. Namely, for any fixed $k$ larger than the critical value

$$
k^{*}=k^{*}(a)=\frac{2 a-2}{2 a-1}
$$

they found the critical value of the parameter $s$ in order to have coexistence.

As observed in $[15,19]$, the similarity of languages facilitates coexistence, the minimal similarity required [see (4)] only depends on $a$ and it is an increasing function of $a$ in the interval $(1,2)$. 
But a question remains: what happens for $a<1$ ? In the pure Abrams-Strogatz model (i.e., the model of the present article for $k=0$ ), stability is possible for $0<a<1$ [21]. For $k \neq 0$, from computer simulations it was observed in [20] that $a<1$ causes that final monolingual situations are not stable and both languages survive (within their respective monolingual groups as well as in the bilingual one) for any values of the parameters $k$ and $s$ and for any initial distribution of speakers. Nevertheless, it was not possible to work out the stability of the equilibrium points in a rigorous way for $a<1$, because the Jacobian matrix is not well defined, with diverging terms. This is done now in the present work. It is worth mentioning at this point that values of $a<1$ could be invoked in the framework of the Abrams-Strogatz model to arrive to configurations of stable coexistence, therefore, this is an added value to explore the regime $a<1$.

The article is organized as follows: in Section 2, we analyze the system for $a \in(0,1)$, in particular, we study the nullclines and the existence of fixed points, whereas in Section 3, we study the stability of the fixed points. Sections 4 and 5 are devoted to the study of the case $a=0$ and $a<0$, respectively. Some remarks and hints for further investigation are contained in the last section.

\section{FIXED POINTS AND NULLCLINES FOR $\boldsymbol{a} \in(\mathbf{0}, 1)$}

We rewrite system (3) using $x+y+b=1$, in the following way:

$$
\begin{aligned}
& \dot{x}=(1-k) s(1-x)(1-y)^{a}-(1-s) x(1-x)^{a}, \\
& \dot{y}=(1-k)(1-s)(1-y)(1-x)^{a}-s y(1-y)^{a},
\end{aligned}
$$

where without loss of generality we have set $c=1$. In this section, we consider the case $a \in(0,1)$. Like in the case $a$ $\in[1,2]$ (see [Colucci et al., submitted]) the set

$$
A=\left\{(x, y) \in R^{2}: \quad x, y \geq 0, x+y \leq 1\right\},
$$

that represents all the possible configurations of the different fractions of speakers, is positively invariant. We observe that for any values of the parameter $k$ and $s$ the system admits at least three fixed points:

$$
P_{1}=(1,1), P_{2}=(1,0), P_{3}=(0,1),
$$

where the point $P_{1}$ is outside the positively invariant region $A$ and the other two points are on $\partial A$.

Whereas in the case $a \geq 1$ by the Jacobian analysis, we get that $P_{2}$ and $P_{3}$ are always stable and $P_{1}$ is always unstable, in this case the Jacobian matrix is not defined at $P_{i}, i=1,2,3$. However, by simply using the equations of the system, when $x=1$ we get that

$$
\dot{x}=0 \rightarrow x(t) \equiv 1,
$$

and

$$
\dot{y}=-s y(1-y)^{a},
$$

we infer that the line $x=1$ is a stable curve for $P_{2}$ and unstable for $P_{1}$. By the same argument, we get that $y=1$ is an unstable curve for $P_{1}$ and stable for $P_{3}$. Then, we conclude that $P_{1}$ is unstable.

We will study indirectly the stability of the fixed points $P_{2}$ and $P_{3}$ by studying the existence and stability of further fixed points (see Section 3 ).

To find further fixed points, we note that the vector field admits linear nullclines, that is, $x=1$ and $y=1$ and hyperbolic nullclines (the $x$-nullclines and $y$-nullclines are the lines on which $\dot{x}=0$ and $\dot{y}=0$ ), respectively):

$$
\left\{\begin{array}{l}
(1-k) s(1-y)^{a}-(1-s) \frac{x}{(1-x)^{1-a}}=0, \\
(1-k)(1-s)(1-x)^{a}-s \frac{y}{(1-y)^{1-a}}=0 .
\end{array}\right.
$$

The hyperbolic nullclines can be written as function of $x$ and $y$, respectively:

$$
\begin{gathered}
n_{1}(x):=1-\left[\frac{(1-s)}{(1-k) s}\right]^{1 / a} \frac{x^{\frac{1}{a}}}{(1-x)^{\frac{1}{a}-1}}, \\
n_{2}(y):=1-\left[\frac{s}{(1-k)(1-s)}\right]^{1 / a} \frac{y^{\frac{1}{a}}}{(1-y)^{\frac{1}{a}-1}} .
\end{gathered}
$$

The intersection of the hyperbolic nullcline can be also written in the following way:

$$
\begin{gathered}
(1-k)^{2}(1-x)(1-y)=x y, \\
(1-s)^{2} x(1-x)^{2 a-1}=s^{2} y(1-y)^{2 a-1} .
\end{gathered}
$$

The hyperbolic nullcline $n_{1}(x)$ satisfies

$$
\lim _{x \rightarrow 1} n_{1}(x)=-\infty, n_{1}(0)=1,
$$

and

$$
n_{1}^{\prime}(x)<0, \forall x \in(0,1), s \in(0,1), k \neq 1 .
$$

As a consequence $n_{1}(x)$ is strictly decreasing. The other nullcline satisfies:

$$
\lim _{y \rightarrow 1} n_{2}(y)=-\infty, n_{2}(0)=1,
$$

and

$$
n^{\prime}{ }_{2}(y)<0, \forall y \in(0,1), s \in(0,1), k \neq 1 \text {. }
$$

Then, the nullcline $n_{2}(y)$ is a strictly decreasing function. Then, also $n_{2}^{-1}(x)$ is strictly decreasing and satisfies $n_{2}^{-1}(1)=0$ (Figure 1). From the properties of the nullclines, as both $n_{1}(x)$ and $n_{2}^{-1}(x)$ are strictly decreasing, and as they do not change concavity (it is a straightforward computation), there exists a unique intersection between them in the set $(0,1) \times(0,1)$ that we call $P$. 


\section{FIGURE 1}
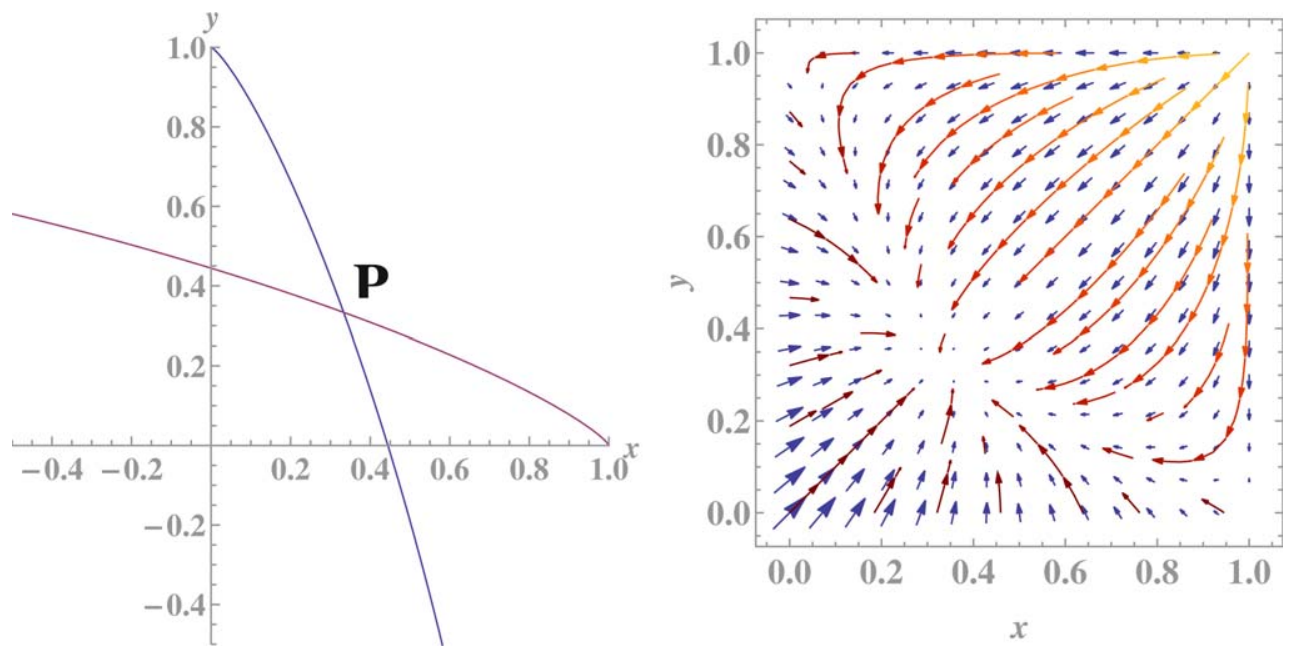

Hyperbolic nullclines and vector field for $a=0.8, k=0.5$, and $s=0.5$. The straight lines $x=1$ and $y=1$ are asymptotes. Due to monotonicity and to the fact that they do not change concavity, they intersect at only one point inside the region $A$, which results to be stable.

To determine the location of the fixed point $P$, we can use the expression (12). We have that $k=1$ then $P=(0,0)$, whereas if we put $k=0$ in (12), we obtain that $P$ is on the line $x+y=1$, between $P_{2}$ and $P_{3}$. Again using (12), we have that

$$
(1-x-y)=x y \frac{k(2-k)}{(1-k)^{2}} \geq 0, \forall x, y \in[0,1]
$$

from which we conclude that if $k, s \neq 0,1$ then $P$ is below the line $x+y=1$ inside the region $A$. Then,

\section{Theorem 2.1}

For all $k, s \in(0,1)$, the system (5) admits the three fixed points $P_{1}, P_{2}, P_{3}$ and the fixed point $P$ inside the region $A$.

\section{Remark 2.2}

We note that if $P$ is stable, then the sytstem (5) admits coexistence solutions of the three populations for $k, s \in(0,1)$.

\section{STABILITY OF THE FIXED POINT $\boldsymbol{P}(\boldsymbol{a} \in(\mathbf{0}, 1))$}

After studying the stability of $P_{1}$, in this section we are doing the same for $P$ and we are also obtaining it for $P_{2}$ and $P_{3}$ in an indirect way. Because $x, y \neq 1$, we have that the Jacobian is always well defined at $P$ together with its trace and determinant. We call the functional Jacobian of the vector field, $\mathcal{A}=\left(a_{i, j}\right)$. In detail, we have:

$$
\begin{aligned}
a_{11} & =-(1-k) s(1-y)^{a}-(1-s)(1-x)^{a}+a(1-s) x(1-x)^{a-1}, \\
a_{12} & =-a s(1-k)(1-x)(1-y)^{a-1}, \\
a_{21} & =-a(1-k)(1-s)(1-y)(1-x)^{a-1}, \\
a_{22} & =-(1-k)(1-s)(1-x)^{a}-s(1-y)^{a}+a s y(1-y)^{a-1} . \\
\operatorname{Det}(\mathcal{A}) & =\left[(1-k)^{2}\left(1-a^{2}\right)+1\right] s(1-s)(1-x)^{a}(1-y)^{a} \\
& +(1-k) s^{2}(1-y)^{2 a-1}[1-y-a y] \\
& +(1-k)(1-s)^{2}(1-x)^{2 a-1}[1-x-a x] \\
& +a(1-s) s(1-x)^{a-1}(1-y)^{a-1}[x(1-y)+y(1-x)+a x y] .
\end{aligned}
$$

Using (12), (13), and the expression of nullclines, we simplify the determinant formula:

$$
\begin{aligned}
\operatorname{Det}(\mathcal{A})= & (1-k)^{2} s(1-s)(1-x)^{a}(1-y)^{a}+s(1-s)(1-x)^{a}(1-y)^{a} \\
& +(a+1)(1-k) s^{2}(1-y)^{2 a}+(a+1)(1-k)(1-s)^{2}(1-x)^{2 a} \\
& -a(1-k)(1-s)^{2} x(1-x)^{2 a-1}-a(1-k) s^{2} y(1-y)^{2 a-1}
\end{aligned}
$$

Let us show that

$$
(1-k)^{2} s(1-s)(1-x)^{a}(1-y)^{a}-a(1-k)(1-s)^{2} x(1-x)^{2 a-1}>0,
$$

and that

$$
\begin{aligned}
& s(1-s)(1-x)^{a}(1-y)^{a}+(a+1)(1-k) s^{2}(1-y)^{2 a} \\
& \quad-a(1-k) s^{2} y(1-y)^{2 a-1}>0 .
\end{aligned}
$$

Using the expression of the hyperbolic nullclines (10), the first inequality can be rewritten as: 


$$
(1-k)(1-s)^{2} x(1-x)^{2 a-1}-a(1-k)(1-s)^{2} x(1-x)^{2 a-1}>0,
$$

and it is verified as $a \in(0,1)$. The second inequality is now:

$$
\begin{aligned}
\frac{1}{1-k} s^{2} y(1-y)^{2 a-1} & +(a+1)(1-k) s^{2}(1-y)^{2 a} \\
-a(1-k) s^{2} y(1-y)^{2 a-1} & >0 .
\end{aligned}
$$

Again, using the expression of the hyperbolic nullclines, we can rewrite the previous inequality as:

$$
\frac{1}{1-k} y+(a+1)(1-k)(1-y)-a(1-k) y>0,
$$

from which

$$
\left(\frac{1}{(1-k)^{2}}-2 a-1\right) y+a+1>0 .
$$

The previous inequality is verified as $a+1>0$ and, from the following inequality,

$$
\frac{1}{(1-k)^{2}}-a>0
$$

We have that $\operatorname{Det}(\mathcal{A})>0$ for all $a \in(0,1)$ and $x, y$ inside $[0,1] \times[0,1]$. We pass on to study the trace:

$$
\begin{aligned}
\operatorname{Tr}(\mathcal{A})= & -(2-k)\left[s(1-y)^{a}+(1-s)(1-x)^{a}\right]+a\left[s y(1-y)^{a-1}\right. \\
& \left.+(1-s) x(1-x)^{a-1}\right] .
\end{aligned}
$$

We simplify the expression of the trace using nullclines:

$$
\operatorname{Tr}(\mathcal{A})=\{a(1-k)-(2-k)\}\left\{s(1-y)^{a}+(1-s)(1-x)^{a}\right\} .
$$

Then, as $a \in(0,1)$, we have that the trace is always negative at $P$. Then, we can conclude:

\section{Theorem 3.1}

For any $a \in(0,1), k, s \in(0,1)$, the system (5) admits the stable fixed point $P$ located inside the region $A$.

Using the same argument of the analysis of the trace of the functional Jacobian, we have that the divergence of the vector field

$$
\begin{aligned}
\operatorname{div}(\mathcal{A})= & -(2-k) s(1-y)^{a}-(2-k)(1-s)(1-x)^{a} \\
& +a(1-s) x(1-x)^{a-1}+\operatorname{asy}(1-y)^{a-1}
\end{aligned}
$$

is always negative inside $[0,1] \times[0,1]$. Then, by the Bendixon theorem, we have:

\section{Theorem 3.2}

There are no periodic orbits inside the region $[0,1] \times[0,1]$.

\section{Remark 3.3}

Since $x=1$ and $y=1$ are stable curves and since on $x=0$ and $y=0$ the vector field is tangent or points inward $A$, we can exclude the existence of periodic orbits which intersect the boundary of $[0,1] \times[0,1]$.

\section{Remark 3.4}

The present model does not admit coexistence of oscillatory type. To recover this possibility, it could be interesting to consider a nonautonomous version of the model with the parameter $s$ replaced by a function of time $s(t)$ (perhaps periodic). It seems reasonable that the relative status of the language may change, however, this process should take place at a different (slower) time scale.

\section{CASE $\boldsymbol{a}=\mathbf{0}$}

If $a=0$, the system (3) becomes:

$$
\begin{gathered}
\dot{x}=(1-k) s-x(1-k s), \\
\dot{y}=(1-k)(1-s)-y(1-k+k s),
\end{gathered}
$$

then, the two equations of the system can be easily solved separately (we note that, for the pure Abrams-Strogatz model, the fractions of the stable populations speaking $X$ and $Y$ are $s$ and $1-s)$. We obtain the fixed point $P=(\bar{x}, \bar{y})$ whose coordinates are

$$
\bar{x}=\frac{s-k s}{1-k s}, \quad \bar{y}=\frac{1-k-s+k s}{1-k+k s} .
$$

The fixed point $P$, if it exists, is stable and it is easy to check that it belongs to the region $A$, as it satisfies $\bar{x}+\bar{y} \leq 1$.

If $k s=1(k=s=1)$, then the $x$-axis is made up of stable fixed points (the orbits are vertical lines), whereas if $1-k+k s=0$, that is $k=1$ and $s=0$, then the $y$-axis is made up of stable fixed points and the orbits are horizontal lines. If $k=1$ and $s \neq\{0,1\}$, then $P=(0,0)$, if $s=1$ and $k \neq 1$ then $P=(1,0)$, whereas if $s=0$ and $k \neq 1$ then $P=(0,1)$. Moreover, we have that:

$$
\begin{aligned}
& \bar{x}=1 \Longleftrightarrow s=1, \\
& \bar{x}=0 \Longleftrightarrow s=0, \text { or } k=1, \\
& \bar{y}=1 \Longleftrightarrow s=0, \text { and } k \neq 1, \\
& \bar{y}=0 \Longleftrightarrow s=1, \text { or } s \neq 0 \text { and } k=1 .
\end{aligned}
$$

Finally we have that $\bar{x}+\bar{y}=1$ if and only if at least one of the following holds

$$
s=1, \quad s=0, \quad k=0 .
$$

From the above analysis, we can conclude that:

\section{Proposition 4.1}

The system (28) admits coexistence solutions for any $k, s \in(0,1)$, that is, there exists a stable fixed point $P$ inside the region $A$. 


\section{FIGURE 2}
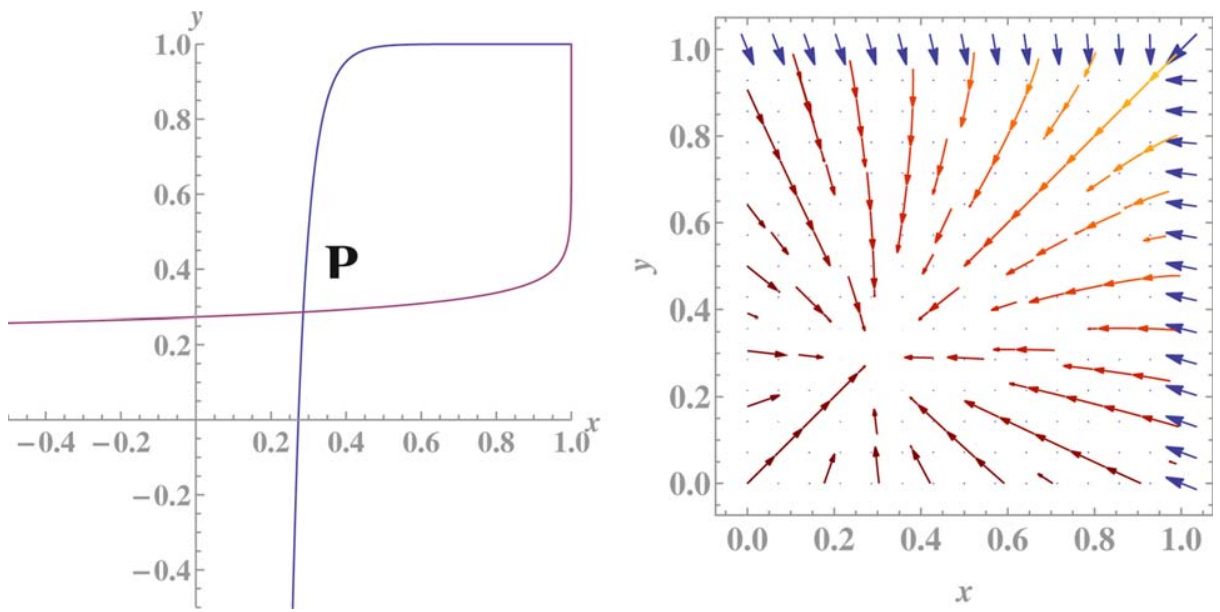

Hyperbolic nullclines and vector field for for $a=-0.2, k=0.6$, and $s=0.5$. The straight lines $x=0$ and $y=0$ are asymptotes. Due to monotonicity and to the fact that they do not change concavity, they intersect at only one point inside the region $A$, which results to be stable.

\section{NeGATiVE VALUES OF $\boldsymbol{a}$}

In this section, we study the case $a<0$. Since for negative values of the exponent $a$, the vector field is not defined if $x=1$ and/or $y=1$, we consider the following set:

$$
\tilde{A}=A \backslash\{(1,0),(0,1)\} .
$$

We claim that the set $\tilde{A}$ is positive invariant, indeed it is possible to use the same argument of the case $a \geq 0$ except for the points $(1,0)$ and $(0,1)$. If starting inside $\tilde{A}$ we approach $(0,1)$, that is, $y(t) \rightarrow 1$, then $\dot{y}(t)$ becomes negative and the solutions can not reach the point $(0,1)$. The same argument works for $(1,0)$.

In this case, the hyperbolic nullclines are strictly increasing (see Figure 2), they do not change concavity and they satisfy

$$
n_{1}(1)=n_{2}^{-1}(1)=1,
$$

and

$$
\lim _{x \rightarrow 0} n_{1}(x)=\lim _{y \rightarrow 0} n_{2}(y)=-\infty
$$

Using again the arguments of Section 2 and (18) we get that, for $a<0$ and $k, s \in(0,1)$, the system (5) admits a unique fixed point $P$ inside $A$.

We recall that the expression of the trace of the Jacobian is

$$
\operatorname{Tr}(\mathcal{A})=\{a(1-k)-(2-k)\}\left\{s(1-y)^{a}+(1-s)(1-x)^{a}\right\},
$$

and we observe that it is always negative when $a<0$. Coming back to the expression (19) of the determinant of
$\mathcal{A}$, we note that it is positive when $a \in[-1,0)$. Then, it remains to study the determinant for $a<-1$.

We consider again the expression of the determinant (19) and simplify it using the expression of the nullclines:

$$
\begin{aligned}
& (1-k)^{2} s(1-s)(1-x)^{a}(1-y)^{a}=(1-k) s^{2} y(1-y)^{2 a-1} \\
& \quad>(a+1)(1-k) s^{2}(1-y)^{2 a},
\end{aligned}
$$

where the last inequality follows from

$$
y>(a+1)(1-y) .
$$

Moreover, using again the nullclines, we have that

$$
\begin{gathered}
s(1-s)(1-x)^{a}(1-y)^{a}=\frac{(1-s)^{2}}{(1-k)} x(1-x)^{2 a-1} \\
>(a+1)(1-k)(1-s)^{2}(1-x)^{2 a},
\end{gathered}
$$

and the last inequality follows as

$$
\frac{x}{(1-k)^{2}}>(a+1)(1-x) \text {. }
$$

From the previous computations, we obtain that the determinant of the Jacobian of the system is always positive inside $\tilde{A}$ and then we can conclude:

\section{Theorem 5.1}

For any $a<0, k, s \in(0,1)$, the system admits a stable fixed point inside the region $\tilde{A}$.

\section{Remark $\mathbf{5 . 2}$}

Using again the Bendixon's Criterion, there are no periodic orbits inside $[0,1] \times[0,1]$. Moreover, since on the lines $x=1$ and $y=1$ the system is not defined and since on 


\section{FIGURE 3}
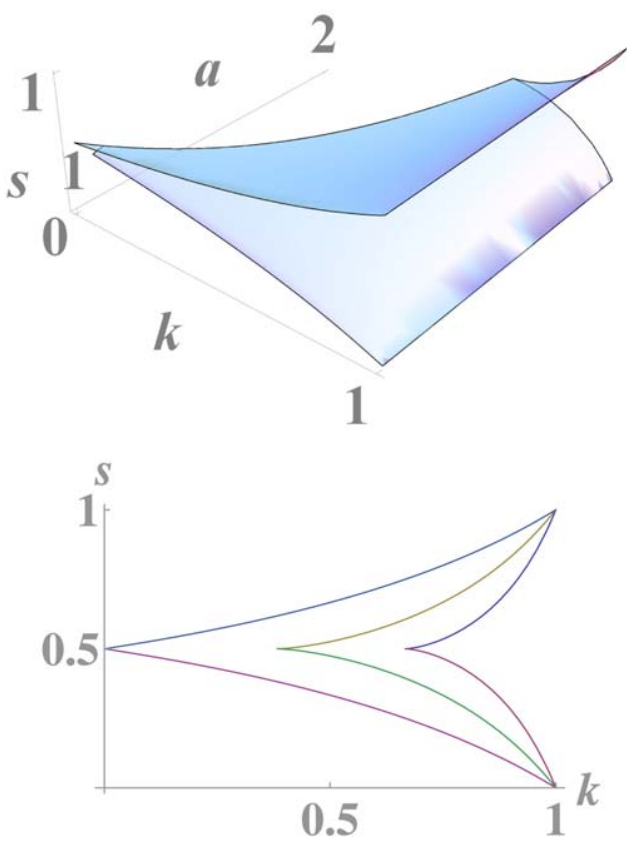

The coexistence region depends on the parameter $(k, s, a)$, in particular its area is decreasing with respect to $a$. In the two figures above, we represent the boundary of the coexistence region. In the first figure, we represent it with a three-dimensional graph for $(k, s, a) \in[0,1] \times[0,1] \times[1,2]$, whereas in the second, we compare the extreme cases $a=1,2$ with the case of Abrams and Strogatz, $a=1.31(a=1,1.31,2$ from left to right. See [Colucci et al., submitted] for the details of the computation of the coexistence region).

$x=0$ and $y=0$ the vector field is tangent or points inward $\tilde{A}$, we can also exclude the existence of periodic orbits that intersect the boundary of $[0,1] \times[0,1]$. As a direct consequence, we obtain the analogous result for $\tilde{A}$.

\section{CONCLUSION}

In this article, we have completed the study of the system (5)-that models language competition-in the case $a<1$, where the exponent $a$ reflects the importance of the size of a group of speakers in attracting new members. So far, fits of real data to the models of Abrams and Strogatz [8] and Mira and Paredes [15, 19] have yielded $a>1$. Nevertheless, it is reasonable to speculate about scenarios with $a<1$. How important is group size in language dynamics? A range $0<a<1$ means that the larger the group the larger the attraction, but with a trend that attenuates when the number of speakers of a given language grows. From the point of view of the individual speaker such situation may make sense: if a community of speakers of language $X$ attains a certain level, a speaker of language $Y$ could not be so sensitive to further increments of the number of speakers of $X$. As shown in this article, it is easier to achieve stable coexistence of both competing languages in the regime $0<a<1$, because the growth of the population of one language is not so aggressive to the other.

Special mention deserves the case $a<0$. Does it have any sense? It might have it. Once $a$ goes below 0 , the size of the group is no longer an attractive factor (the attraction decreases when the size of a given group increases). Under this circumstance, minorities would become stronger, reproducing cases of minorities that become stronger when they feel under menace of extinction, for example. In parallel, majorities would become less aggressive, prompted by a perception of invulnerability. $a<0$ describes also the case of rejecting attitudes against the group to which the speaker belongs to, when such group is the dominant one.

In any case, it seems reasonable to think that the power of attraction of a monolingual group could change to a saturation regime (in that moment, the group size does not play any role, $a=0$ ). This, combined with a perception of risk by minorities, making them to reduce their probability to change to the dominant language, would move the regime to cases with $a<0$. In general, by a simple analogy with predator-prey models, whenever the system approaches the stable points $(1,0)$ or $(0,1)$ (i.e., extinction of any of the languages), the exponent $a$ could change from $a>1$ to $0<a$ $<1$ and even to $a<0$. This points out that, to understand better what is going on in these limiting cases, it could be useful to open a room for a nonconstant $a$ exponent.

As a conclusion, the whole picture of the coexistence problem for the model (5) has been obtained for the parameters that satisfy the following conditions

$$
a \leq 2, \quad k \in[0,1], \quad s \in[0,1] .
$$

For $a \in[1,2]$, the coexistence region in the plane $(k, s)$ has a curvilinear triangular shape with vertexes in the points $(1,0),(0,1)$, and $\left(k^{*}(a), 1 / 2\right)$ where (see [Colucci et al., submitted])

$$
k^{*}(a)=\frac{2 a-2}{2 a-1} .
$$

As observed in $[15,19]$, the similarity of languages facilitates coexistence, the minimal similarity required [see (4)] only depends on $a$. We note that if $a=1$ (see [22] for a detailed study for $k, s \in(0,1)$ ) then $k^{*}=0$ whereas for $a=2$ we have that $k^{*}=2 / 3$ (see Figure 3).

This means that a higher value of the parameter $a$ needs a higher level of similarity between the languages in order to obtain coexistence. Conversely, if $a$ is close to 1 , the requested similarity is near zero. These arguments show that the model (5) can describe cases of coexistence even if the similarity between the languages is low or is zero. 
In particular, if $(a, k, s)=\left(1,0, \frac{1}{2}\right)$, we have a segment $(x+y=1, x, y \in[0,1])$ of stable fixed points connecting the points $P_{2}$ and $P_{3}$. Any solution starting inside $A$ converges to a point of the segment, except the extremes $P_{2}$ and $P_{3}$. Then, we have coexistence of the two monolingual groups and extinction of the bilingual group. This means that the change from language $X$ to $Y$ is compensated by the opposite change, whereas there is no interest in the bilingual group.

We have also shown that, in the cases in which $a<1$, any solution starting inside $A$ arrives to a point of stable coexistence. The case $a=1$ represents a limit case: it shares both features of the case $a<1$ and $a \in(1,2]$. Whenever $a$ passes the critical value 1 , the similarity parameter gains importance; but, if $a=1$, we have the interesting case $(k, s)=(0,1 / 2)$; that is, if the status of the two languages is the same, then the similarity is not relevant.

Finally, we have also shown that, for any value of the parameter $a$, there always exist coexistence solutions, which makes the model (5) a realistic tool to describe real cases in which two languages compete and coexist. As a consequence, the qualitative behavior of the model is not affected by possible noise in determining the parameters of the system.

\section{REFERENCES}

1. Miller, G.A. The Science of Words; Scientific American Library: New York, 1996.

2. Sole, R.V.; Corominas-Murtra, B.; Valverde, S.; Steels, L. Language networks: Their structure, function, and evolution. Complexity 2010, 15, 20-26.

3. Castellano, C.; Fortunato, S.; Loreto, V. Statistical physics of social dynamics. Rev Mod Phys 2009, 81, 591-646.

4. Febres, G.; Jaffé, K.; Gershenson, C. Complexity measurement of natural and artificial languages. Complexity, 2014, doi: $10.1002 /$ cplx.21529.

5. Searls, D.B. The language of genes. Nature 2002, 420, 211-217.

6. Eroglu, S. Language-like behavior of proteins length distribution in proteomes. Complexity, 2014, doi: 10.1002/cplx.21498.

7. Epstein, J.M. Agent-based computational models and generative social science. Complexity 1999, 4, 41-60.

8. Abrams, D.M.; Strogatz, S.H. Modelling the dynamics of language death. Nature 2003, 424, 900.

9. Solé, R.V.; Corominas-Murtra, B.; Fortuny, J. Diversity, competition, extinction: The ecophysics of language change. J R Soc Interface 2010, 7, 1647-1664.

10. Crystal, D.; Language Death; Cambridge University Press: Cambridge, 2000.

11. Nettle, D.; Romaine, S. Vanishing Voices: The Extinction of the World's Languages, 1st ed.; Oxford University Press: New York, 2000.

12. Minett, J.W.; Wang, W.S.-Y. Modelling endangered languages: The effects of bilingualism and social structure. Lingua 2008, $118,19-45$.

13. Heinsalu, E.; Patriarca, M.; Léonard, J.L. The role of bilinguals in language competition. Adv Complex Syst 2014, 17, 1450003, doi: 10.1142/S0219525914500039.

14. Kabatek, J. Modelos matemáticos e substitución lingüística. Estud Linguist Galega 2012, 4, 27-43.

15. Mira, J.; Paredes, A. Interlinguistic similarity and language death dynamics. Europhys Lett 2005, 69, 1031-1034.

16. Chapel, L.; Castelló, X.; Bernard, C.; Deffuant, G.; Eguíluz, V.M.; Martin, S.; San Miguel, M. Viability and resilience of languages in competition. PLoS ONE 2011, 5, e8681.

17. Pinasco, J. P.; Romanelli, L. Coexistence of languages is possible. Physica A 2006, 361, 355-360.

18. Vogt, V. Modeling interactions between language evolution and demography. Hum Biol 2009, 81, 237-258.

19. Mira, J.; Seoane, L.F; Nieto, J.J. The importance of interlinguistic similarity and stable bilingualism when two languages compete. New J Phys 2011, 13, pp. 9, Art. 033007.

20. Otero-Espinar, M.V.; Seoane, L.F.; Nieto, J.J.; Mira, J. Analytic solution of a model of language competition with bilingualism and interlinguistic similarity. Physica D 2013, 264, 17-26.

21. Patriarca, M.; Castelló, X.; Uriarte, J.R.; Eguíluz, V.M.; San Miguel, M. Modeling two-language competition dynamics. Adv Complex Syst 2012, 15, 1250048.

22. Nie, L.; Teng, Z.; Nieto, J.J.; Jung, I.H. Dynamic analysis of a two languages competitive model with control strategies. Math Probl Eng 2013, 2013, pp. 13, Article ID 654619. 